Portland State University

PDXScholar

Chemistry Faculty Publications and

Presentations

Chemistry

$9-22-2017$

\title{
Toxicant Formation in Dabbing: The Terpene Story
}

Jiries Meehan-Attrash

Portland State University

Wentai Luo

Portland State University

Robert M. Strongin

Portland State University, strongin@pdx.edu

Follow this and additional works at: https://pdxscholar.library.pdx.edu/chem_fac

Part of the Medicinal-Pharmaceutical Chemistry Commons

Let us know how access to this document benefits you.

\section{Citation Details}

Meehan-Atrash, J., Luo, W., \& Strongin, R. M. (2017). Toxicant Formation in Dabbing: The Terpene Story. ACS omega, 2(9), 6112-6117.

This Article is brought to you for free and open access. It has been accepted for inclusion in Chemistry Faculty Publications and Presentations by an authorized administrator of PDXScholar. Please contact us if we can make this document more accessible: pdxscholar@pdx.edu. 


\title{
Toxicant Formation in Dabbing: The Terpene Story
}

\author{
Jiries Meehan-Atrash, Wentai Luo, and Robert M. Strongin*(0) \\ Department of Chemistry, Portland State University, 1719 SW 10th Avenue, Portland, Oregon 97201, United States
}

\section{Supporting Information}

ABSTRACT: Inhalable, noncombustible cannabis products are playing a central role in the expansion of the medical and recreational use of cannabis. In particular, the practice of "dabbing" with butane hash oil has emerged with great popularity in states that have legalized cannabis. Despite their growing popularity, the degradation product profiles of these new products have not been extensively investigated. The study herein focuses on the chemistry of myrcene and other common terpenes found in cannabis extracts. Methacrolein, benzene, and several other products of concern to human health were formed under the conditions that simulated realworld dabbing. The terpene degradation products observed are consistent with those reported in the atmospheric chemistry literature.

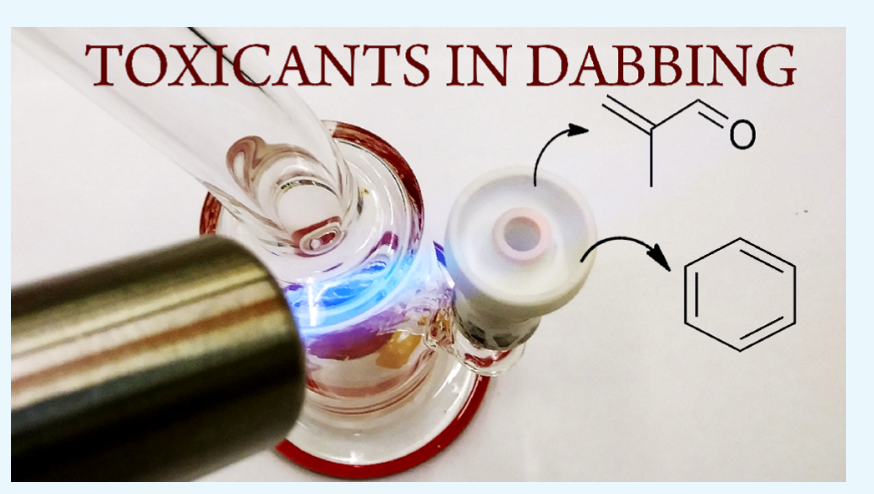

\section{INTRODUCTION}

Terpenes and terpenoids are present in such a wide diversity of environments (nature, food, cosmetics, pharmaceuticals, and drugs) that their consequences for inhalation toxicology cannot be ignored. Additionally, their inclusion in flavored electronic cigarettes $^{1}$ and ubiquitous presence in inhalable cannabis products are of particular concern. The medicinal and psychoactive effects of cannabis have been proposed to be enhanced by terpenes, a phenomenon known as the "entourage effect", 2 and these relatively unsubstantiated assertions of benefits have led the cannabis industry to place a heavy emphasis on these aroma compounds.

Terpenoid degradation in the context of cannabis has not been extensively studied; ${ }^{3,4}$ however, it has attracted attention in the context of atmospheric chemistry. ${ }^{5,6}$ For instance, the reactions of terpenoids with $\mathrm{O}_{3}$ and $\mathrm{NO}_{x}$ are well-known, but they are not directly applicable to e-cigarettes or inhalable cannabis products. However, these and other studies of pyrolysis and combustion of terpenoids should serve as a starting point toward understanding the reaction pathways in consumer vaporization devices. Despite the growing popularity of flavored e-cigarettes and terpene-enriched cannabis extracts, the chemical profiles of their terpene degradation products have not been evaluated in detail.

Of very recent concern is the practice of dabbing, which has emerged as a dangerous and rapidly growing trend in cannabis consumption. It consists of inhaling the vapors produced by placing a small amount of cannabis extract ( $\mathrm{a}$ "dab") on a small heated surface (the "nail"), which is connected to a water pipe. ${ }^{7}$ Its delivery of harmfully large amounts cannabinoids 8,9 represents a potential danger to consumers, but little is known about the toxicants the process may produce.
The principal extract used in dabbing is butane hash oil (BHO). BHO is a resinous, nonpolar extract of the cannabis made using butane as a solvent. ${ }^{10} \mathrm{BHO}$ has active ingredient (tetrahydrocannabinol (THC) or cannabidiol) contents ranging between 50 and $90 \%,{ }^{8,11}$ with terpene content ranging from 0.1 to $34 \%$ (unpublished). Myrcene is unequivocally the most abundant terpene in cannabis, followed by limonene, linalool, pinene, caryophyllene, and humulene; however, the plant can contain up to 68 additional terpenic compounds in trace amounts. ${ }^{12}$ Additionally, some consumers increase the terpenoid content by dipping $\mathrm{BHO}$ in a vial of terpenes prior to use ("terp dipping").

BHO is made by passing butane over cannabis buds and leaves, and subsequently "purging" the butane from the product under vacuum at room temperature or in an oven. Different nuances in its processing can lead to slightly different consistencies, which take on terms such as shatter, budder, crumble, pull-and-snap, wax, and so on. In all of its forms, the extract is a sticky, resinous substance similar to the oleo-resins of other plants. ${ }^{14}$ Because the process does not involve heating the extract to the point that delta-9-tetrahydrocannabinolic acid (THCA, the native form of this substance found in the plant) decarboxylates (unpublished) into the active THC, BHO is not orally active and must be vaporized for the users to achieve its effects. $^{15}$

BHO production started out as a dangerous "backyardchemist" style operation that is famous for causing numerous explosions and house fires. Through the course of legalization, the production has steadily gained sophistication. The most

Received: August 3, 2017

Accepted: September 12, 2017

Published: September 22, 2017 
modern, legal extraction laboratories live up to the OSHA standards with full ventilation and butane recovery. Modern techniques also include steps to "de-wax" the product by dissolving the crude $\mathrm{BHO}$ in isopropyl alcohol and chilling in a freezer, and, finally, filtering off the precipitated waxes in a process known as winterization. Many subtleties in its production exist, but many remain secretive due to the highly competitive nature of the cannabis marketplace and the general inability of extract producers to file patents due to the drug's legal status at the federal level.

In addition to butane extraction, supercritical $\mathrm{CO}_{2}$ extraction has gained traction due to the fact that is does not leave any trace of hydrocarbon solvents in the end product. ${ }^{16}$ The cannabis extract made by this method, colloquially known as $\mathrm{CO}_{2}$ oil, has a lesser viscosity than $\mathrm{BHO}$, a property that allows it to be used in vaporizer pens on its own with no cutting agents. The lesser viscosity is due to the fact that the supercritical extraction process requires the product to be first decarboxylated (heating in an oven at $\left.100+{ }^{\circ} \mathrm{C}\right),{ }^{17}$ leaving an extract consisting of all THC (an oil at room temperature) and no THCA (a solid at room temperature). $\mathrm{CO}_{2}$ oil is generally more expensive than $\mathrm{BHO}$ and mostly present on the market in prefilled vaporizer cartridges and not commonly as a standalone extract for dabbing. Because this extraction method does not leave residual hydrocarbons, it has been named, along with alcohol extracts, as the only allowable medical extracts to be sold under the medical cannabis regulations in New York, ${ }^{18}$ Minnesota, Ohio, and Pennsylvania.

According to a recent survey, ${ }^{11}$ the main reasons for using dabs are that less material is needed to get the desired effect and a "cleaner high." Consumers consider dabbing to be a form of vaporization, and, therefore, view it as easier on the lungs than smoking. ${ }^{19}$ However, little information exists on the prevalence of dabbing. From 213 BHO extraction laboratories in the 17 states raided in 2014, 2015 saw a steep increase in the number of laboratories raided to 337 in 26 states. ${ }^{20}$ An analysis of the Twitter content related to dabs found a greater popularity in the states that have legalized recreational and/or medical cannabis. $^{21}$

Different types of nails, the surface on which vaporization occurs, exist on the market. Use of an electrically controlled nail ("e-nail") allows temperature control; but, more commonly, users heat the nail (made of titanium, ceramic, or quartz) with a crème brulee torch ${ }^{22}$ and have no temperature control. A minority of dabbers use lower temperatures to preserve flavor, whereas a majority use higher temperatures to assure complete vaporization with no wasted material. E-nail users posting online cite a preferred temperature around $710{ }^{\circ} \mathrm{F}\left(378{ }^{\circ} \mathrm{C}\right)$, but cite a range from $340-482{ }^{\circ} \mathrm{C} .{ }^{23-25}$ Raber et al. reported a dabbing temperature of $300{ }^{\circ} \mathrm{C}$, but this was only an (low) estimate. The boiling point of THC has recently been predicted to be ca. $417^{\circ} \mathrm{C},{ }^{26}$ but vaporization can occur at temperatures lower than this by the use of a "carb cap" that reduces pressure on its surface during inhalation. ${ }^{27}$

This study is an initial effort toward assessing the safety of dabbing cannabis extracts. Due to the fact that these consist of a complex mixture, we have begun our focus on terpenoids, the component we predict to be the most thermally labile. To study dabbing, we carefully recreated the inhalation topography and temperatures employed by users. The study described herein is the first to investigate the degradation products from dabbing and is focused on the terpene fraction of the extracts used by consumers.

\section{RESULTS AND DISCUSSION}

Sample Generation and Product Identification. We investigated the dabbing temperature ranges (TRs, Figure 1)

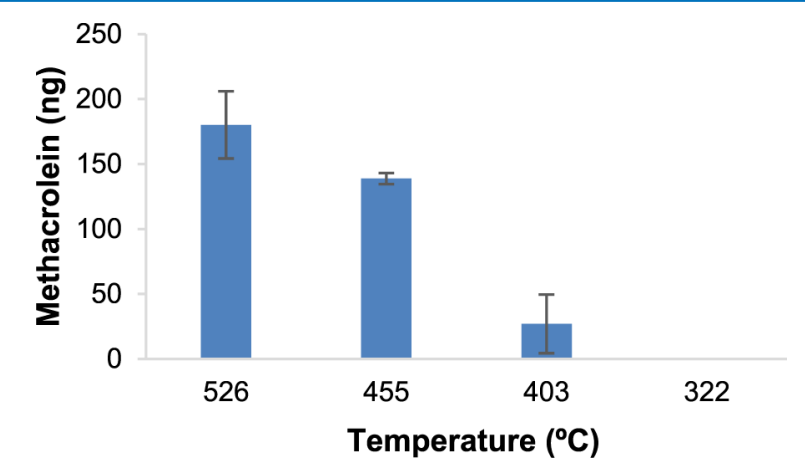

Figure 1. MC (ng) generated in a $40 \mathrm{mg}$ dab using myrcene as a model terpene assuming a $5.9 \%$ concentration of terpenes in $\mathrm{BHO}$. Temperature values represent the $T_{\mathrm{m}}$ for each $\mathrm{TR}$. Error bars are determined at the $95 \%$ confidence level using the standard deviation of the three replicates taken at each TR. At the lowest TR, MC was not detected by NMR.

inclusive of and beyond the ranges of those reported by the users. The vapor collection and analysis methods were based on those by Jensen et al. $^{28}$ using an impinger filled with NMR solvent for vapor collection. In the dabbing simulation experiments herein, the vapor generated from the heated ceramic nail connected to a water pipe passed through a cold trap followed by the impinger. The impinger was, in turn, connected to a smoking machine that generated the airflow. Degradation products from myrcene, limonene, linalool, and Fire OG cannabis terpenes, a commercially available mix specifically fabricated for terp dipping, were monitored. ${ }^{11}$ The presence of methacrolein (MC) and benzene in vapor NMR samples was confirmed by spiking with authentic samples (Supporting Information). Their levels were quantified by NMR using an internal standard.

In addition to the NMR method, the dabbing vapor was collected using an adsorption/thermal desorption (ATD) cartridge and analyzed using an automated adsorption/thermal desorption-gas chromatography-mass spectrometry (ATDGC-MS) method similar to that in Pankow et al. ${ }^{29}$ Additional product structures (Scheme 1) were assigned by the GC-MS analysis. Other minor products that have been previously described in the literature ${ }^{30}$ were also tentatively identified in the chromatographs (Supporting Information). Air blanks were collected and analyzed using each of the NMR and the ATDGC-MS methods.

Temperatures in dabbing experiments were carefully monitored for consistency using a thermographic camera. As the first drop in terpene touched the nail, an initial temperature $\left(T_{\mathrm{i}}\right)$ was recorded. Once a $10 \mathrm{~s}$ draw concluded, a final temperature $\left(T_{\mathrm{f}}\right)$ was recorded (the nail cooled between 50 and $30{ }^{\circ} \mathrm{C}$ during the draw due to convection). A median temperature $\left(T_{\mathrm{m}}\right)$ was calculated and averaged for each replicate to afford a representative $T_{\mathrm{m}}$ for each TR.

The ${ }^{1} \mathrm{H}$ NMR spectra from the dabbing samples displayed peaks characteristic of a range of organic acid, aldehyde, and aromatic products. The two products appearing in high abundance in the spectra were the toxins benzene and MC (Scheme 1, Table 1). MC is a well-known degradation product of isoprene, ${ }^{5,31,32}$ which is itself a known degradation product 
Scheme 1. Terpene Degradation Products Identified via the GC-MS Analysis ${ }^{a}$

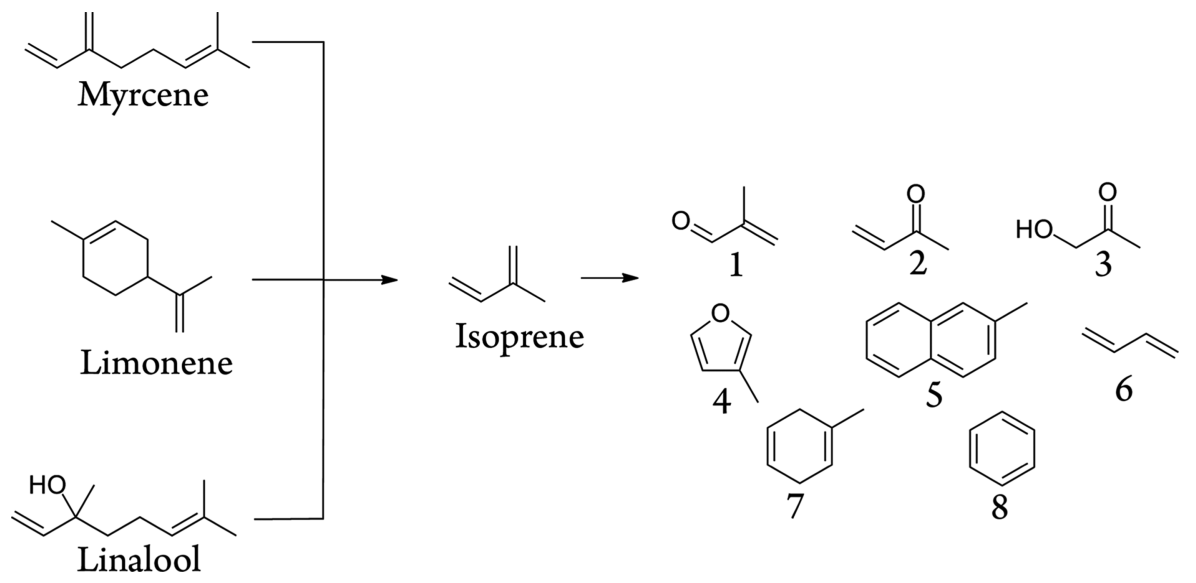

${ }^{a}$, Methacrolein; 2, methyl vinyl ketone; 3, hydroxyacetone; 4, 3-methylfuran; 5, 2-methylnapthalene; 6, 1,3-butadiene; 7, 1-methylcyclohexa-1,4diene; 8 , benzene. These and other related products were produced from pure samples of each of limonene, linalool, and myrcene.

Table 1. Methacrolein (MC) and Benzene Levels Produced per mg Terpene Starting Material When Vaporized at the Highest Temperature Range Investigated, ca. $550{ }^{\circ} \mathrm{C}\left(T_{\mathrm{i}}\right)$ $-500{ }^{\circ} \mathrm{C}\left(T_{\mathrm{f}}\right)$ Using Single Replicate Experiments

\begin{tabular}{lcc} 
& MC (ng/mg terpene) & benzene $(\mathrm{ng} / \mathrm{mg}$ terpene $)$ \\
"Fire OG" & 127 & 10 \\
limonene & 261 & 63 \\
linalool & 103 & $\mathrm{ND}$ \\
myrcene & 81 & 60 \\
\hline
\end{tabular}

of myrcene ${ }^{33}$ and other terpenes. ${ }^{34}$ Benzene, alkyl benzenes, and polycyclic aromatic hydrocarbons are known to form during terpene thermolysis. For example, benzene has been observed as a degradation product in the synthesis of myrcene by the pyrolysis of $\beta$-pinene, ${ }^{35}$ and it is also a product of solanesol pyrolysis. ${ }^{34}$ Benzene has also been detected in cannabis smoke. ${ }^{36}$

Product Quantification. Given the wide diversity of the terpenes present in $\mathrm{BHO}$, the relatively high abundance of myrcene and the similarity of the products from each of the terpenes studied (Table 1 and Scheme 1), we focused on myrcene as a model terpene in evaluating the effect of temperature on the yields of MC and benzene. Assuming 40 $\mathrm{mg}$ as an average size $\mathrm{dab},{ }^{22}$ each dab contains $2.36 \mathrm{mg}$ of terpenes, which is based on an average concentration of terpenes of $5.9 \%$ in $\mathrm{BHO}$ (unpublished data). The amount of MC obtained per dab based on these calculations is displayed in Figure 1.

Because dabbing topography has not been previously investigated, we chose an inhalation volume of $338 \mathrm{~mL}$ and a $10 \mathrm{~s}$ duration to assure a more complete collection of vapor. The concentrations of $\mathrm{MC}$ in ppb per dab in this regime are $185 \pm 11 \mathrm{ppb}$ at $T_{\mathrm{m}}=526{ }^{\circ} \mathrm{C}, 157 \pm 2 \mathrm{ppb}$ at $T_{\mathrm{m}}=455^{\circ} \mathrm{C}$, $131 \pm 9 \mathrm{ppb}$ at $T_{\mathrm{m}}=403{ }^{\circ} \mathrm{C}$, and undetectable at $T_{\mathrm{m}}=322^{\circ} \mathrm{C}$.

Benzene was not detected below the highest TR. Using the same rationale as above for $\mathrm{MC}$ emission, one dab of $\mathrm{BHO}$ delivers $17 \mathrm{ng}$ of benzene. Represented as a concentration in the draw volume, this value is $15 \pm 1.8 \mathrm{ppb}$.

Degradant Toxicology. MC's property as a noxious irritant is unsurprising due to its structural similarity to acrolein, a powerful pulmonary irritant ${ }^{37}$ and an air pollutant of great concern. Ambient concentrations of MC outside of
Stockholm were determined to be $0.06 \mathrm{ppb}$, whereas those at different urban locations in Stockholm were 0.11, 0.13, 0.19, and $0.71 \mathrm{ppb}^{38} \mathrm{MC}$ 's effect on the respiratory tract in mice has shown it to be a potent irritant, indicating its threshold limit value should not exceed $0.3 \mathrm{ppm} .{ }^{39}$ Nøjgaard et al. reported changes in the blink frequency during eye exposure to $\mathrm{MC}$ at a concentration of $100 \mathrm{ppb}$ and proposed a LOEL of $286 \mathrm{ppb}^{40}$ These conflicting reports indicate that the safe levels of MC are yet to be determined.

Unlike MC, the toxicology of benzene has been thoroughly evaluated. Although benzene is a ubiquitous pollutant, the concentrations of benzene found in the dabbing terpenes at the highest TR are far greater than those found in ambient air. The average concentration of benzene, a potent carcinogen, in U.S. air, measured over 137 different sites is $0.313 \mathrm{ppb}$ (313 ppt), ${ }^{3,41}$ and is correspondingly the "largest single known cancer-risk air toxic (sic)." ${ }^{22}$

Degradant Formation Mechanism. We propose that the formation of $\mathrm{MC}$ and benzene occurs via isoprene as an intermediate (Scheme 1). The GC-MS spectra of limonene, linalool, and myrcene all displayed significant peaks tentatively assigned to isoprene, which suggests that these terpenes, the major terpenes in $\mathrm{BHO}$, break down to their isoprene monomers before further degradation.

Studies of the atmospheric chemistry of isoprene have shown that it reacts with hydroxyl radicals and $\mathrm{O}_{2}$ to form not only $\mathrm{MC}$ and $\mathrm{HCHO}$ but also methyl vinyl ketone and 3methylfuran. The GC-MS analysis of each pure terpene studied afforded a tentative identification with a high match quality of MC, methyl vinyl ketone, and 3-methylfuran, as well as 1,3-butadiene and several cyclic and acyclic dienes, polyenes, and aromatics (Scheme 1 and Supporting Information).

Limitations. The main limitation of this study is the fact that the concentrations of MC and benzene determined are likely underestimated. One reason may be the relatively large draw volume used. In addition, the temperature-dependent concentration values were extrapolated from myrcene, which afforded the lowest yield of degradation products of all of the terpenes investigated. Another factor potentially contributing to the underestimation of yields is transfer inefficiency resulting in the potential losses of terpenes and their products. For example, the average myrcene recovery $(8.7 \pm 0.7 \mathrm{mg})$ was low compared to the amount delivered onto the nail $(59.6 \mathrm{mg})$. 
Although this low yield of terpenes in the NMR sample was initially attributed to their limited solubility in DMSO- $d_{6}$, dabbing experiments using $\mathrm{CDCl}_{3}$ also had low yield by NMR. This may not be due entirely to degradation. Transfer inefficiency in dabbing has been previously described. ${ }^{22}$

\section{CONCLUSIONS}

Given the widespread legalization of cannabis in the United States, it is imperative to study the full toxicology of its consumption to guide future policy. The results of these studies clearly indicate that dabbing, although considered a form of vaporization, may in fact deliver significant amounts of toxic degradation products. The difficulty users find in controlling the nail temperature put users at risk of exposing themselves to not only methacrolein but also benzene. Additionally, the heavy focus on terpenes as additives seen as of late in the cannabis industry is of great concern due to the oxidative liability of these compounds when heated. This research also has significant implications for flavored e-cigarette products due to the extensive use of terpenes as flavorings. Future research will also be directed toward assessing the contribution of terpenoids to the existing toxicant formation in e-cigarettes. Additionally, the methods discussed herein will also be used to further study the degradation of cannabis extracts used in dabbing and cannabis e-cigarettes.

\section{METHODS}

Materials. Terpenes included myrcene $\geq 95 \%$, stabilized, FCC, FG (Sigma-Aldrich); (R)-(+)-limonene analytical standard (Sigma-Aldrich); linalool $\geq 97 \%$, FCC, FG; and Fire OG terpene mix (Blue River Extracts).

NMR Experiments. Air is drawn at a constant rate using and the Single Cigarette Smoking Machine (SCSM-STEP, CH Technologies) calibrated to pull $338 \mathrm{~mL}$ air during a $10 \mathrm{~s}$ dab. A HIVE Domeless Element $10 \mathrm{~mm}$ ceramic nail (HIVE Ceramics) was attached to a small dab water pipe (Zion Cannabis in Portland, OR). For each separate experiment, the water pipe was filled with $20 \mathrm{~mL}$ of fresh $200 \mathrm{ppm}$ solution of $\mathrm{NaCl}$ Biological, Certified Crystalline (Fisher Scientific) in HPLC grade water (Honeywell).

Terpene $(15 \mu \mathrm{L})$ was delivered per dab using a Hamilton 50 $\mu \mathrm{L}$ analytical syringe. Five dabs were done per experiment. The vapor was collected through a cold trap chilled with isopropyl alcohol/dry ice at $-77{ }^{\circ} \mathrm{C}$, proceeded by an impinger containing $750 \mu \mathrm{L}$ of DMSO- $d_{6}+0.05 \% \mathrm{v} / \mathrm{v}$ tetramethylsilane (99.9\%, Cambridge Isotope). After the experiment was concluded, the cold trap was washed with the NMR solvent in the impinger and collected quantitatively using an Eppendorf P1000 pipette in an NMR tube. The water pipe and the cold trap were connected by $5 \mathrm{~cm}$ of $1 / 2 \mathrm{in}$. outer diameter ACF0027-F Tygon S3 E-3603. The end connected to the water pipe was wrapped in Teflon tape to make it fit snugly. The cold trap and the impinger were connected by $3.5 \mathrm{~cm}$ of $1 / 2$ in. outer diameter ACF0027-F Tygon S3 E-3603. The impinger and the SCSM were connected by $5 \mathrm{~cm}$ of $3 / 8$ in. outer diameter ACF0017-F Tygon S3 E-3603. The tubing was discarded after every experiment, so sorptive losses were consistent with every experiment.

All of the NMR samples were spiked with $10 \mu \mathrm{L}$ of a 17.33 $\mathrm{mM}$ solution of 2,3,5,6-tetrachloronitrobenzene (TCI Chemicals) in DMSO- $d_{6}$ using an Eppendorf P10 pipette. This standard solution was made by adding $11.23 \mathrm{mg}$ of 2,3,5,6tetrachloronitrobenzene to $3 \mathrm{~mL}$ of DMSO- $d_{6}$.

Myrcene dab NMR experiments at each TR (Figure 1) were performed in triplicate. Terpene experiments shown in Table 1 were performed once each. The exact conditions used in recording the NMR spectra are presented in the SI.

ATD-GC-MS. The same water pipe (containing $20 \mathrm{~mL} 200$ ppm solution of $\mathrm{NaCl}$ ) and the same ceramic nail were connected to an ATD cartridge with $5 \mathrm{~cm}$ of $1 / 2$ in. outer diameter ACF0027-F Tygon S3 E-3603 wrapped in the Teflon tape to make a seal and then attached to $5 \mathrm{~cm}$ of $3.5 \mathrm{~cm}$ of $3 / 8$ in. outer diameter ACF0027-F Tygon S3 E-3603, also wrapped with Teflon tape on the end to assure an air-tight seal. The other end of the ATD cartridge was connected to the SCSMSTEP using $5 \mathrm{~cm}$ of $3.5 \mathrm{~cm}$ of $3 / 8 \mathrm{in}$. outer diameter ACF0027F Tygon S3 E-3603. The ATD cartridges used contained 100 $\mathrm{mg}$ of 35/60 mesh Tenax TA and $200 \mathrm{mg}$ of 60/80 mesh Carbograph 1 TD (Camsco Inc., Houston, TX). The same dabbing topography used in the NMR experiments were used in the ATD cartridge sample collections. This high flow rate exceeds that normally used for these cartridges, but this was allowed due to the fact that these experiments were only used for product identification and not quantification. The conditions used in the ATD cartridge analysis are explained in the SI.

Thermography. The temperature of the nail was acquired real time by the infrared thermography using an FLIR T450sc 2.0 (FLIR Systems). The emissivity of the ceramic nail was determined experimentally to be 0.9 by comparing it to the known value of insulating electrical tape, 0.97 . The reflected temperature was determined by the reflector method to be 23 ${ }^{\circ} \mathrm{C}$. The TRs used in the NMR experiments are shown in Figure 1, and the TR used in the ATD-GC-MS experiment was the second hottest TR with a $T_{\mathrm{m}}$ of $455{ }^{\circ} \mathrm{C}$. All of the temperature data for each experiment are presented in the SI.

\section{ASSOCIATED CONTENT}

\section{S Supporting Information}

The Supporting Information is available free of charge on the ACS Publications website at DOI: 10.1021/acsomega.7b01130.

Experimental conditions, materials, and characterization data for MC and benzene (PDF)

\section{AUTHOR INFORMATION}

\section{Corresponding Author}

*E-mail: strongin@pdx.edu.

ORCID

Robert M. Strongin: 0000-0003-3777-8492

\section{Author Contributions}

J.M.-A. performed all of the experiments, collected sample, and wrote the manuscript. R.M.S. supervised the studies and edited the manuscript. W.L. ran the ATD-GC-MS samples, advised on sample collection by this method, and reviewed the manuscript. All of the authors have given approval to the final version of the manuscript.

\section{Funding}

We thank the NIH and FDA for support of this work via award R01ES025257. The content is solely the responsibility of the authors and does not necessarily represent the views of the $\mathrm{NIH}$ or the FDA. 


\section{Notes}

The authors declare no competing financial interest.

\section{ACKNOWLEDGMENTS}

Dr. Jorge Escobedo provided the guidance and useful discussion in the experiments and during manuscript drafting. Dr. David Peyton provided the guidance and useful discussion in the NMR methods used. Tim Frasca reviewed the manuscript for content and style.

\section{ABBREVIATIONS}

MC, methacrolein; THC, tetrahydrocannabinol; CBD, cannabidiol; $\mathrm{BHO}$, butane hash oil; TR, temperature range; $\mathrm{HCHO}$, formaldehyde; TLV, threshold limit value; LOEL, lowest observed effect level

\section{REFERENCES}

(1) Tierney, P. A.; Karpinski, C. D.; Brown, J. E.; Luo, W.; Pankow, J. F. Flavour chemicals in electronic cigarette fluids. Tob. Control 2016, 25, e10-e15.

(2) Russo, E. B. Taming THC: potential cannabis synergy and phytocannabinoid-terpenoid entourage effects. Br. J. Pharmacol. 2011, 163, 1344-1364.

(3) do Monte, D. S.; Tenório, J. A. B.; Bastos, I. V. G. A.; Mendonça, F. d. S.; Neto, J. E.; da Silva, T. G.; Ramos, C. S. Chemical and biological studies of beta-carotene after exposure to Cannabis sativa smoke. Toxicol. Rep. 2016, 3, 516-522.

(4) Iqbal, M. S.; Khan, M. U. A.; Akbar, J.; Shad, M. A.; Masih, R.; Chaudhary, M. T. Isoconversional thermal and pyrolytic GC-MS analysis of street samples of hashish. J. Anal. Appl. Pyrolysis 2016, 122, $175-182$.

(5) Atkinson, R. Gas Phase Tropospheric Chemistry of Organic Compounds: A Review. Atmos. Environ., Part A 1989, 24, 1-41.

(6) Calogirou, A.; Larsen, B. R.; Kotzias, D. Gas-phase terpene oxidation products: a review. Atmos. Environ. 1999, 33, 1423-1439.

(7) Stogner, J. M.; Miller, B. L. The Dabbing Dilemma: A Call for Research on Butane Hash Oil and Other Alternate Forms of Cannabis Use. Subst. Abuse 2015, 36, 393-395.

(8) Pierre, J. M.; Gandal, M.; Son, M. Cannabis-induced psychosis associated with high potency "wax dabs". Schizophr. Res. 2016, 172, 211-212.

(9) Murray, R. M.; Quigley, H.; Quattrone, D.; Englund, A.; Di Forti, M. Traditional marijuana, high-potency cannabis and synthetic cannabinoids: increasing risk for psychosis. World Psychiatry 2016, 15, 195-204.

(10) Stogner, J. M.; Miller, B. L. Assessing the Dangers of "Dabbing": Mere Marijuana or Harmful New Trend? Pediatrics 2015, 136, 1-3.

(11) Loflin, M.; Earleywine, M. A new method of cannabis ingestion: the dangers of dabs? Addict. Behav. 2014, 39, 1430-1433.

(12) Ross, S. A.; ElSohly, M. A. The volatile oil composition of fresh and air-dried buds of Cannabis sativa. J. Nat. Prod. 1996, 59, 49-51.

(13) danner1620 Terp Dippin' Kosher Kush Rosin with Blue River Extracts [video file]. https://www.youtube.com/watch?v=YOFkbcMoso (accessed July 7, 2017).

(14) Stogner, J. M.; Miller, B. L. The Dabbing Dilemma: A Call for Research on Butane Hash Oil and Other Alternate Forms of Cannabis Use. Subst. Abuse 2015, 36, 393-395.

(15) ElSohly, M. A. Marijuana and the Cannabinoids, 1st ed.; Humana Press: NJ, 2007.

(16) Perrotin-Brunel, H.; Perez, P. C.; van Roosmalen, M. J. E.; van Spronsen, J.; Witkamp, G. J.; Peters, C. J. Solubility of Delta(9)tetrahydrocannabinol in supercritical carbon dioxide: Experiments and modeling. J. Supercrit. Fluids 2010, 52, 6-10.

(17) Perrotin-Brunel, H.; van Roosmalen, M. J. E.; van Spronsen, J.; Verpoorte, R.; Peters, C. J.; Witkamp, G. J. Supercritical fluid extraction of cannabis: experiments and modelling of the process design. ISASF-Graz 2010, 1-6.
(18) Manufacturing Requirements for Approved Medical Marihuana Products. Official Compilation of Codes, Rules and Regulations of the State of New York, Part 1004, Section 11(b), Title 10, 2017.

(19) Gieringer, D.; St. Laurent, J.; Goodrich, S. Cannabis Vaporizer Combines Efficient Delivery of THC with Effective Suppression of Pyrolytic Compounds. J. Cannabis Ther. 2004, 4, 7-27.

(20) 2015 National Drug Threat Assessment Summary; Report Number DEA-DCT-DIR008-16; US Department of Justice, United States Drug Enforcement Agency; Homeland Security Digital Library, 2015.

(21) Daniulaityte, R.; Nahhas, R. W.; Wijeratne, S.; Carlson, R. G.; Lamy, F. R.; Martins, S. S.; Boyer, E. W.; Smith, G. A.; Sheth, A. "Time for dabs": Analyzing Twitter data on marijuana concentrates across the U.S. Drug Alcohol Depend. 2015, 155, 307-311.

(22) Raber, J. C.; Elzinga, S.; Kaplan, C. Understanding dabs: contamination concerns of cannabis concentrates and cannabinoid transfer during the act of dabbing. J. Toxicol. Sci. 2015, 40, 797-803.

(23) Burns, D. What's Your Preferred Enail Temp? https://www. reddit.com/r/CannabisExtracts/comments/30mj24/whats_your_ preferred_enail_temp/ (accessed July 18, 2017).

(24) MFletcher, I. Is There a Consensus Temp for Low Temps Dabs on an Enail? https://www.reddit.com/r/enail/comments/4gjznx/is there_a_consensus_temp_for_low_temp_dabs_on_an/ (accessed July 18,2017$)$.

(25) Propanekush What Temperature are you Dabbing at? What Type of Nail? https://www.reddit.com/r/CannabisExtracts/ comments/3onqt $8 /$ what_temperature_are_you_dabbing_at_what type_of/ (accessed July 18, 2017).

(26) Tara, M.; Lovestead, T. J. B. Determination of Cannabinoid Vapor Pressures to Aid in Vapor Phase Detection of Intoxication. Forensic Chem. 2017, 5, 79-85.

(27) Black, S. Why Carb Cap? Elevate Your Dabbing. http:// hightimes.com/culture/why-carb-cap-elevate-your-dabbing/ (accessed July 18,2016$)$.

(28) Jensen, R. P.; Strongin, R. M.; Peyton, D. H. Solvent Chemistry in the Electronic Cigarette Reaction Vessel. Sci. Rep. 2017, 7, No. 42549.

(29) Pankow, J. F.; Kim, K.; McWhirter, K. J.; Luo, W.; Escobedo, J. O.; Strongin, R. M.; Duell, A. K.; Peyton, D. H. Benzene formation in electronic cigarettes. PLoS One 2017, 12, No. e0173055.

(30) McGraw, G. W.; Hemingway, R. W.; Ingram, L. L.; Canady, C. S.; McGraw, W. B. Thermal degradation of terpenes: Camphene, Delta(3)-carene, limonene, and alpha-terpinene. Environ. Sci. Technol. 1999, 33, 4029-4033.

(31) Carter, W. P. L. Condensed atmospheric photooxidation mechanisms for isoprene. Atmos. Environ. 1996, 30, 4275-4290.

(32) Palen, E. J.; Allen, D. T.; Pandis, S. N.; Paulson, S. E.; Seinfeld, J. H.; Flagan, R. C. Fourier Transform Infrared Analysis of Aerosol Formed in the Photo-Oxidation of Isoprene and [beta]-pinene. Atmos. Environ., Part A 1992, 26, 1239-1251.

(33) Egloff, G.; Herrman, M.; Levinson, B. L.; Dull, M. F. Thermal reactions of terpene hydrocarbons. Chem. Rev. 1934, 14, 287-383.

(34) Britt, P. F.; Buchanan, A. C., III; Owens, C. V., Jr. Mechanistic Investigation into the Formation of Polycyclic Aromatic Hydrocarbons from the Pyrolysis of Terpenes. Prepr. Pap.-Am. Chem. Soc., Div. Fuel Chem. 2004, 49, 868-871.

(35) Kolicheski, M. B.; Cocco, L. C.; Mitchell, D. A.; Kaminske, M. Synthesis of myrcene by pyrolysis of [beta]-pinene: Analysis of decomposition reactions. J. Anal. Appl. Pyrolysis 2007, 80, 92-100.

(36) Moir, D.; Rickert, W. S.; Levasseur, G.; Larose, Y.; Maertens, R.; White, P.; Desjardins, S. A comparison of mainstream and sidestream marijuana and tobacco cigarette smoke produced under two machine smoking conditions. Chem. Res. Toxicol. 2008, 21, 494-502.

(37) Wang, H. T.; Hu, Y.; Tong, D.; Huang, J.; Gu, L.; Wu, X. R.; Chung, F. L.; Li, G. M.; Tang, M. S. Effect of carcinogenic acrolein on DNA repair and mutagenic susceptibility. J. Biol. Chem. 2012, 287, 12379-12386.

(38) Jonsson, A.; Persson, K. A.; Grigoriadis, V. Measurements of Some Low Molecular-Weight Oxygenated, Aromatic, and Chlorinated 
Hydrocarbons in Ambient Air and in Vehicle Emissions. Environ. Int. 1985, 11, 383-392.

(39) Larsen, S. T.; Nielsen, G. D. Effects of methacrolein on the respiratory tract in mice. Toxicol. Lett. 2000, 114, 197-202.

(40) Nøjgaard, J. K.; Christensen, K. B.; Wolkoff, P. The effect on human eye blink frequency of exposure to limonene oxidation products and methacrolein. Toxicol. Lett. 2005, 156, 241-251.

(41) EPA's Report on the Environment; Ambient Concentrations of Selected Air Toxics. Exhibit 3; US Environmental Protection Agency, 2014.

(42) George, B. J.; Schultz, B. D.; Palma, T.; Vette, A. F.; Whitaker, D. A.; Williams, R. W. An evaluation of EPA's National-Scale Air Toxics Assessment (NATA): Comparison with benzene measurements in Detroit, Michigan. Atmos. Environ. 2011, 45, 3301-3308. 